\title{
Product Code Optimization for Determinate State LDPC Decoding in Robust Image Transmission
}

\author{
Nikolaos Thomos, Member, IEEE, Nikolaos V. Boulgouris, Member, IEEE, and Michael G. Strintzis, Fellow, IEEE
}

\begin{abstract}
We propose a novel scheme for error-resilient image transmission. The proposed scheme employs a product coder consisting of low-density parity check (LDPC) codes and Reed-Solomon codes in order to deal effectively with bit errors. The efficiency of the proposed scheme is based on the exploitation of determinate symbols in Tanner graph decoding of LDPC codes and a novel product code optimization technique based on error estimation. Experimental evaluation demonstrates the superiority of the proposed system in comparison to recent state-of-the-art techniques for image transmission.
\end{abstract}

Index Terms-Image transmission, low-density parity check (LDPC) codes, product codes, unequal error protection (UEP), wireless transmission.

\section{INTRODUCTION}

$\mathbf{T}$ HE widespread deployment of wireline/wireless communication systems and the proliferation of digital media created the recent surge in multimedia transmission research. Since all communication channels suffer from errors, error-resilient schemes have been developed in order to reliably transmit multimedia over unreliable channels. Most of these systems employ embedded scalable source coders, like the set partitioning in hierarchical trees (SPIHT) [1] and the JPEG2000 [2], for image transmission, and H.264 [3] for video transmission. Forward error correction (FEC) is used in conjunction with rate allocation algorithms to protect effectively the information and guarantee satisfactory image/video quality.

A variety of error-resilient techniques for transmission of images over error-prone channels have been recently proposed in the literature. In [4], a scheme for robust transmission of SPIHT streams over wireless and memoryless channels was presented. Product codes using concatenated rate compatible punctured convolutional codes/cyclic redundancy check codes (RCPC/CRC) [5] and Reed-Solomon (RS) [6] codes were employed for channel error protection. An optimal channel rate allocation algorithm for application with embedded source coders was proposed in [7]. This scheme was optimal not only for transmission at the designed transmission rate, but also

Manuscript received April 7, 2005; revised October 22, 2005. This work was supported in part by the European Commission under Contract FP6-511568 3DTV. The associate editor coordinating the review of this manuscript and approving it for publication was Prof. Yucel Altunbasak.

N. Thomos and M. G. Strintzis are with the Informatics and Telematics Institute (CERTH) and the Electrical and Computer Engineering Department, University of Thessaloniki, GR-541 24 Thessaloniki, Greece (e-mail: nthomos@iti.gr; strintzi@eng.auth.gr).

N. V. Boulgouris is with the Department of Electronic Engineering, Division of Engineering, King's College, London WC2R 2LS, U.K. (e-mail: nikolaos. boulgouris@kcl.ac.uk).

Digital Object Identifier 10.1109/TIP.2006.877433 at the intermediate rates. In [8], a low-complexity algorithm for optimal rate allocation based on ideas initially explored in [7] was presented. An error-resilient scheme for transmission of embedded block-based coders was proposed in [9]. This scheme partitions the bitstream into a number of independently decodable sub-bitstreams which are unequally protected. This approach allowed the decoding after the occurence of uncorrectable errors.

In [10], irregular repeat accumulate (IRA) codes [11] were proposed for the transmission of JPEG2000 and SPIHT streams over binary symmetric channels (BSC). The scheme in [10] showed performance superior to that of the method in [12]. The transmission of SPIHT streams over BSC channels was also considered in [13]. IRA codes with inherent unequal error protection (UEP) capabilities were used for reliable communication. The length of the employed IRA codes was equal to the total transmission rate. A product coding scheme based on recursive convolutional codes (RSC) for transmission of SPIHT streams over BSC and Gilbert-Elliot (GE) channels was presented in [14]. This product coder employed a modified Viterbi algorithm (VA) and tried to eliminate transmission errors by using an iterative decoding process.

In this paper, we propose a novel image transmission methodology based on a product coder consisting of low-density parity check (LDPC) codes [15] and RS codes. The information recovered during each product decoding iteration is considered as a posteriori knowledge and is used for the correction of remaining errors by appropriately modifying the sum-product algorithm (SPA). Interleaving is used in order to further improve the efficiency of iterative decoding. A novel UEP approach is proposed which optimizes transmission by calculating error estimates in iterative product decoding. The novel optimization strategy will be shown to yield considerable performance improvements in comparison to other methods for image transmission.

This paper is arranged as follows. The product coder employed by the proposed scheme and the iterative decoding procedure is described in Section II. The modifications on LDPC decoding algorithm are presented in Section III. The proposed novel channel rate allocation technique is described in Section IV. In Section V, experimental results are reported. Finally, conclusions are drawn in Section VI.

\section{Iterative Decoding of Product Codes BASED ON LDPC AND RS}

The proposed product coder is schematically depicted in Fig. 1. It consists of systematic LDPC codes in the horizontal direction and RS codes in the vertical direction. The outer RS 


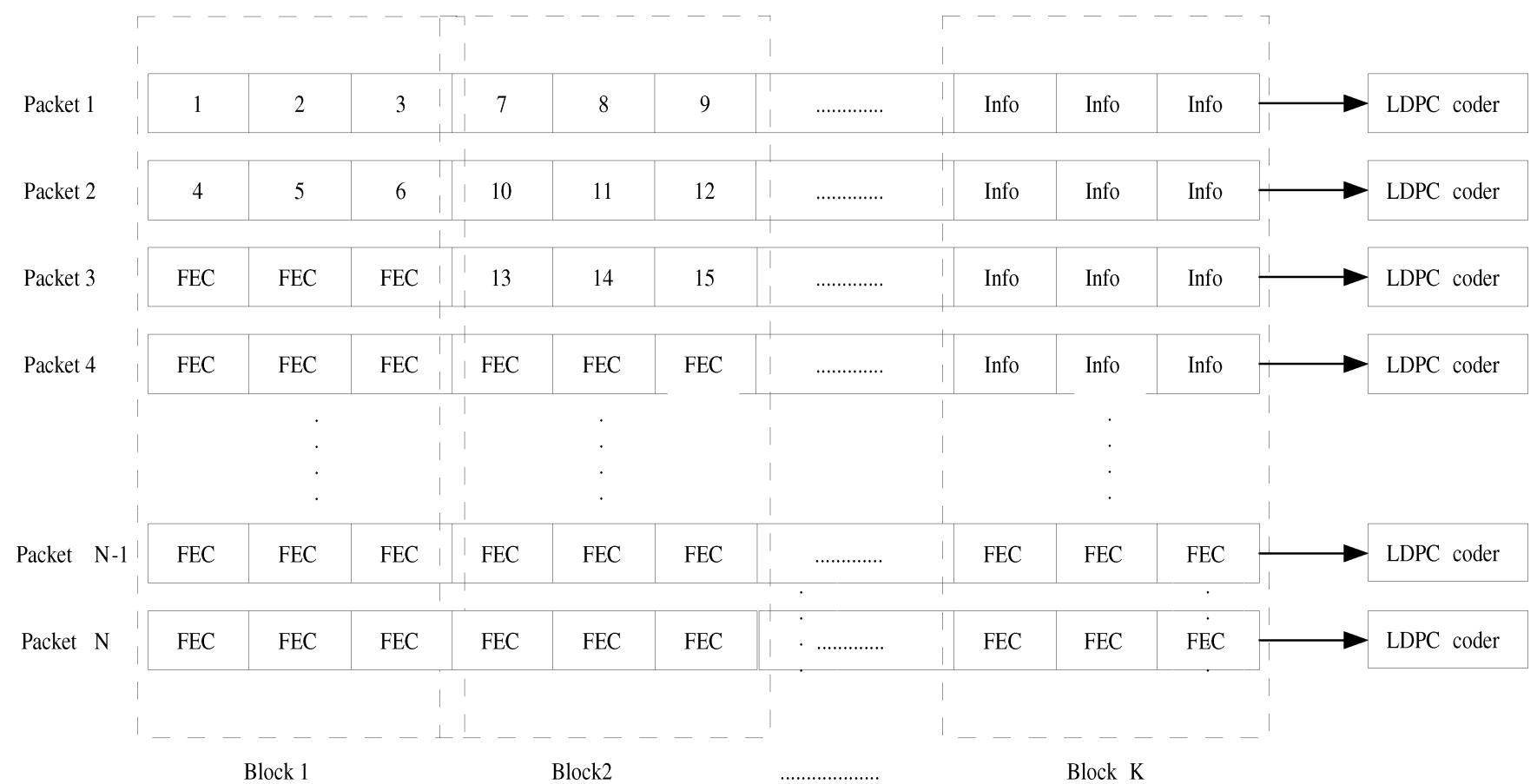

Fig. 1. Product code based on LDPC and RS codes.

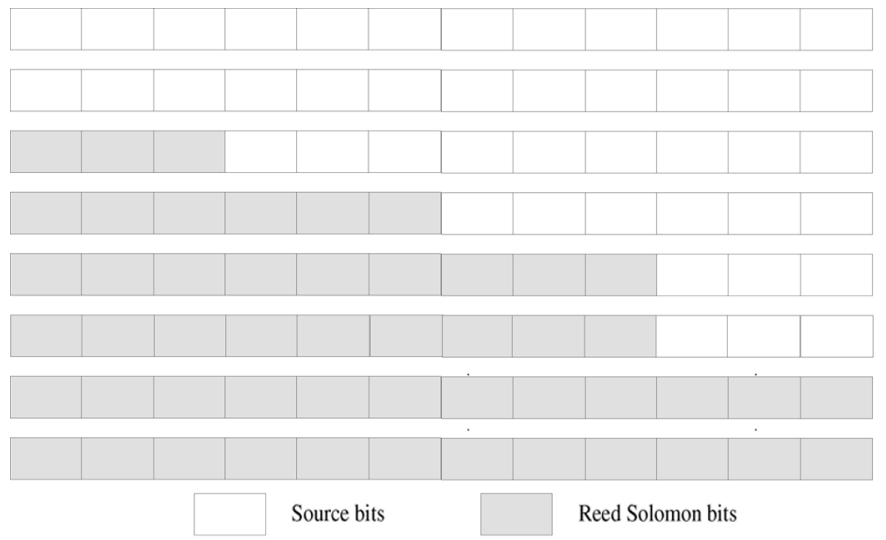

(a)

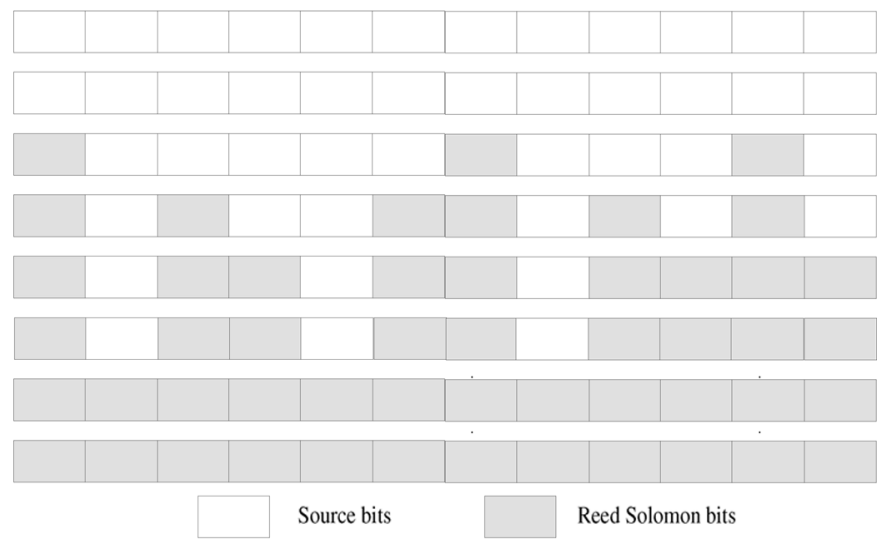

(b)

Fig. 2. Conventional structure of (a) is converted to block with interleaved columns in (b). If the symbols in the most protected columns are always fully recovered, they can serve as determinate information during the subsequent LDPC decoding.

codes are used as erasure correcting codes. Due to the progressively decreasing importance of the source information in blocks $1,2, \ldots, k, \ldots, K$ of Fig. 1 , the number of RS symbols in the $k_{t h}$ block decreases with increasing $k$. The inner LDPC codes cope with random errors. The LDPC codes are also used at the decoder in order to indicate packet erasures if the packet bitstream contains uncorrectable errors after the maximum number of LDPC decoding iterations.

The encoding procedure works as follows: first RS encoding is performed using an UEP strategy. The resulting bitstreams are subsequently interleaved using the S-random interleaver [16] in the horizontal direction. This means that each codeword (row of the product-code array) in Fig. 1 is interleaved using the $\mathrm{S}$-random interleaver in order to randomize errors. The interleaved rows are then LDPC encoded. The initial arrangement of data in a product-code array and the resulting arrangement after interleaving are presented in Fig. 2. At the receiver side, the opposite procedure is followed in an iterative manner.

During product decoding, the LDPC decoding of packets corrects bit errors when it is possible, otherwise indicates the erased packets. Then deinterleaving restores the initial arrangement of data in the product-code array. Right after LDPC decoding and deinterleaving, RS decoding takes place in the vertical direction in order to correct the remaining errors. Due to the UEP, the RS decoding will not, in general, correct all errors in a packet, which means that several packets will be only partially recovered. For this reason, we re-decode the LDPC codes in the horizontal direction by taking into account the fact that, due to the preceding RS decoding stage, some bits are deterministically known to be correct [14], [17]. This additional knowledge boosts the LDPC decoder and enables the correct decoding of some packets that were initially considered erased. This procedure is repeated until 


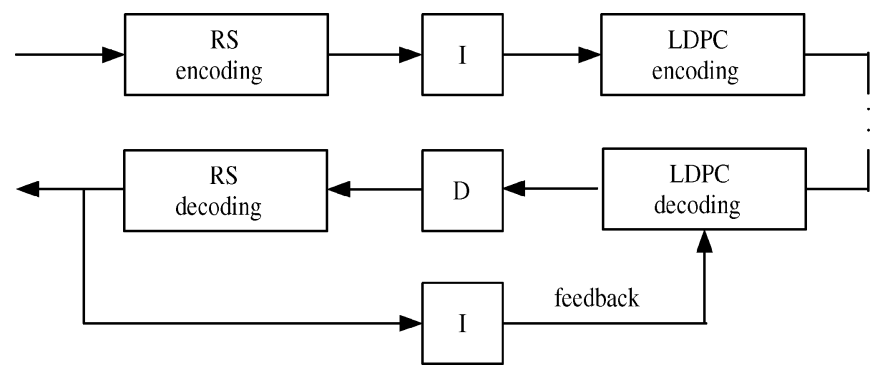

Fig. 3. Encoding and decoding using the proposed scheme. RS-decoded information is fed back to the LDPC decoding module. Interleaving (I) and deinterleaving (D) is performed in order to distribute the determinate bits evenly.

no new segment of information is recovered after a product decoding iteration. ${ }^{1}$ A simplified block diagram of the above encoding and decoding processes is depicted in Fig. 3. Note that, due to the interleaving that is performed after RS decoding in the feedback loop, the LDPC decoding is applied on corrupted packets in which the determinate bits are evenly distributed.

\section{EXPLoitATION OF DETERMINATE SyMbOLS IN LDPC DECODING}

In the previous section, the iterative decoding of the LDPC/RS product-code scheme was presented. The product coder first attempts to correct all errors using LDPC decoding. Whenever this is not possible, a packet erasure is indicated. The blocks in which the erased packets do not exceed the block's RS protection capability are recovered after RS decoding. Therefore, the portions of the bitstream corresponding to the above blocks are error free. The recovery of a bitstream portion after a product decoding iteration benefits the subsequent reapplication of LDPC decoding since fewer bits are erroneous and the knowledge that some specific bits are error free can be used in order to manipulate the soft information exchanged during LDPC decoding iterations.

In order to exploit the deterministic information, we appropriately modified the log-domain version of the SPA, that is usually deployed for LDPC decoding. In general, bipartite graphs called Tanner graphs [18] are used for the representation of LDPC codes. Two types of nodes exist in Tanner graphs: the variable and the check nodes. The SPA algorithm is based on the computation of the a posteriori probability that the value $c_{i}$ of the bit at the $i_{t h}$ position of the transmitted codeword $\underline{c}=\left[\begin{array}{llll}c_{1} & c_{2} & \ldots & c_{n}\end{array}\right]$

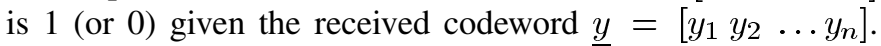
Thus, the log-likelihood ratios are computed by the following equations:

$$
\begin{aligned}
L\left(c_{i}\right) & =\log \left(\frac{\operatorname{Pr}\left(c_{i}=0 \mid \underline{y}\right)}{\operatorname{Pr}\left(c_{i}=1 \mid \underline{y}\right)}\right) \\
L\left(q_{i j}\right) & =\log \left(\frac{q_{i j}(0)}{q_{i j}(1)}\right) \\
L\left(r_{j i}\right) & =\log \left(\frac{r_{j i}(0)}{r_{j i}(1)}\right)
\end{aligned}
$$

where $q_{i j}(b)$ is the extrinsic information passed at the first half of an iteration of LDPC decoding from the variable node $i$ to

${ }^{1}$ Note that there are two types of iterations. A product decoding iteration involves LDPC decoding and RS decoding, while LDPC iterations are part of LDPC decoding. the check node $j$ and $b \in\{0,1\}$. Respectively, $r_{j i}(b)$ is defined as the extrinsic message passed at the second half of an LDPC iteration from the check node $j$ to the variable node $i$.

Since, in general, it is difficult to determine exactly the value of the likelihood ratios, we modify the SPA algorithm by biasing the ratios whenever determinate information is available. This means that whenever the correct value of $b$ is known, we enforce the likelihood ratios in (1)-(3) to take the appropriate sign (determined by $b$ ). In practice, we set the likelihood ratio to be equal to a very large positive value, in case $b$ is equal to 0 , or we set it to a very large negative value if $b$ is equal to 1 . This biasing approach is implicity assumed in the analysis that follows.

The decision regarding bit values in LDPC decoding are taken using the log-likelihood ratios $L\left(Q_{i}\right)$, where [19]

$$
L\left(Q_{i}\right)=L\left(c_{i}\right)+\sum_{j \in C_{i}} L\left(r_{j i}\right) .
$$

If the transmitted bit at the $i_{t h}$ position $c_{i}$ is determinate and takes the value 1 , then it should be $L\left(Q_{i}\right)<0$. Equivalently, the knowledge that the transmitted bit at the $i_{t h}$ position is 1 means that the following relation should hold

$$
L\left(c_{i}\right)+\sum_{j \in C_{i}} L\left(r_{j i}\right)<0 .
$$

The term $L\left(c_{i}\right)$ is determined by the channel and the transmitted bit at the $i_{t h}$ position. In an errorless environment, $L\left(c_{i}\right)$ and $L\left(Q_{i}\right)$ should always have the same sign. Thus, whenever $c_{i}$ is determinate, we enforce $L\left(c_{i}\right)$ to have the same sign as $L\left(Q_{i}\right)$. To further improve the decoding performance we set $L\left(r_{j i}\right)<0$ for all $j \in C_{i}$. In a similar manner, we are biasing $L\left(q_{i j}\right)<0$ when $c_{i}=1$. The application of the above approach to the case in which the value of the determinate bit is equal to 0 is trivial. This manipulation of the soft information that is exchanged between nodes in LDPC iterations has a very beneficial impact on the efficiency of LDPC decoding.

\section{Novel Channel Rate Allocation BASED ON ERROR ESTIMATES}

The occurrence of determinate bits depend on the initial RS-based UEP that is applied in the product code array. The UEP algorithm that we initially used with the present product coding method was based on dynamic programming and was originally proposed in [17] for the transmission of SPIHT streams over wireless channels. This scheme will be hereafter termed as APC since UEP optimization is based on the a priori channel conditions. The amount of protection allocated to data varies in the vertical direction (columns) of a data sub-array which is termed a "block" (see Fig. 1). The optimization process adjusts the amount of RS protection according to the importance of source information. Therefore, more channel symbols (RS symbols) are allocated to blocks carrying important information and fewer to other blocks. In this way, blocks that contribute with higher distortion improvement to the eventual image quality are better protected than the rest. In the following, we first briefly present the APC approach 


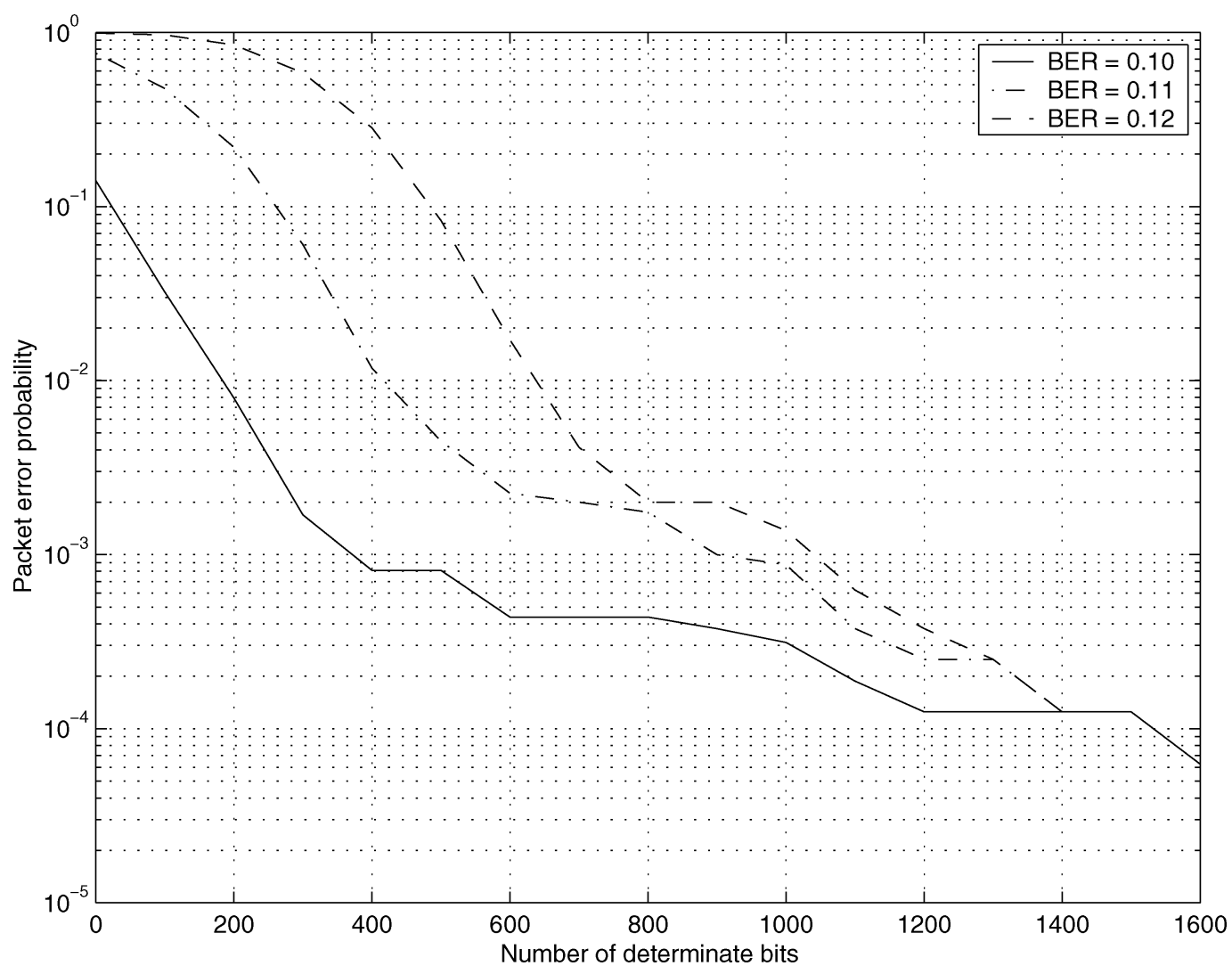

Fig. 4. Packet erasure probabilities of the $(1904,4096)$ LDPC code for variable bit error rates. 50 LDPC iterations were assumed.

and then we focus on the proposed approach for channel rate allocation based on error estimates.

Since RS codes are used, we define the probability $P_{L}(k)$ of losing the $k_{t h}$ block, i.e., the probability that the RS protection, which is allocated to the $k_{t h}$ block is not sufficient to correct the corrupted information in the erased packets. Trivially

$$
\begin{aligned}
P_{L}(k) & =\operatorname{Prob}\{\text { erased packets }>Q(k)\}= \\
& =\sum_{i=Q(k)+1}^{N}\left(\begin{array}{c}
N \\
i
\end{array}\right) \cdot p^{i} \cdot(1-p)^{N-i}
\end{aligned}
$$

where $p$ is the packet erasure probability, $Q(k)$ denotes the number of RS symbols in each column of the $k_{t h}$ block, and $N$ is the number of packets.

We also define

$$
P_{d}(k)= \begin{cases}P_{L}(k), & \text { if } k=1 \\ P_{L}(k)-P_{L}(k-1), & \text { otherwise. }\end{cases}
$$

The expected distortion $\bar{D}$ is given by

$$
\bar{D}=\sigma^{2} \cdot P_{d}(1)+\sum_{k=2}^{K} D_{k-1} \cdot P_{d}(k)+\left(1-P_{L}(K)\right) \cdot D_{K}
$$

where $D_{k}$ is the distortion after the successful decoding of blocks $1, \ldots, k$, and $\sigma^{2}$ is the variance of the pixel intensities. In the following, $k$ is the stage (block) index. The minimization of $\bar{D}$ takes place using dynamic programming.

Although the APC approach will be shown to perform well, it does not take into consideration the iterative product decoding process. Therefore, we would like to optimize the RS rate allocation process, so that the RS decoding provides the optimal number of determinate bits to the LDPC decoder. To this end, we modify the APC technique in order to exploit the determinate bits recovered after a product code iteration. This scheme will be referred to as $A E C$ since adaptively estimated channel conditions are considered during optimizations. The AEC approach differs to APC in that the packet loss probabilities are updated on the fly during optimization, based on the number of available determinate bits (see Fig. 4), while, in the APC technique, the original error probabilities remain unaltered.

Next, we briefly summarize the proposed optimization algorithm which is based on dynamic programming. A trellis is constructed in which the number of states is equal to the number of available RS protection levels and the number of stages equals the number of the blocks to be protected (see Fig. 1). Our objective is to minimize the total expected distortion for the transmission of blocks $1, \ldots, K$, where $K$ is the total number of transmitted blocks. To this end, we define the adaptively calculated probability $\tilde{P}_{L}(k)$ of losing the $k_{t h}$ block when the AEC approach is followed. Specifically

$$
\begin{aligned}
\tilde{P}_{L}(k) & =\text { Prob }\{\text { erased packets }>Q(k)\}= \\
& =\sum_{i=Q(k)+1}^{N}\left(\begin{array}{c}
N \\
i
\end{array}\right) \cdot p_{k}^{i} \cdot\left(1-p_{k}\right)^{N-i}
\end{aligned}
$$

where $p_{k}$ is the packet erasure probability considering that the bits in the first $k-1$ blocks are determinate (see Fig. 4). 
TABLE I

PSNR COMPARISONS OF THE PROPOSED SCHEMES FOR THE TRANSMISSION OF “LENA" FOR TRANSMISSION OVER BSC WITH BER $=0.1$

\begin{tabular}{|c||c|c|c|c|c|c|}
\hline \multicolumn{1}{|c||}{ Rate } & \multicolumn{2}{c|}{ UEP } & \multicolumn{2}{c|}{ APC } & \multicolumn{2}{c|}{ AEC } \\
\hline (in bpp) & $(3880,8272)$ & $(1904,4136)$ & $(3880,8272)$ & $(1904,4136)$ & $(3880,8272)$ & $(1904,4136)$ \\
\hline 0.252 & 29.48 & 29.48 & 29.70 & 29.68 & 30.73 & 30.61 \\
\hline 0.505 & 32.54 & 32.70 & 32.65 & 32.77 & 33.77 & 33.62 \\
\hline 0.994 & 35.80 & 35.85 & 36.00 & 35.98 & 36.80 & 36.69 \\
\hline
\end{tabular}

Using the above definitions, the optimization algorithm used with AEC can be summarized as follows.

1. Set $k=1$ and $\bar{D}_{0}=\sigma^{2} \cdot \tilde{P}_{L}(1)$.

2. For each trellis node in the $k_{t h}$ stage, ${ }^{2}$ i.e., for all possible protection choices for the $k$ th block, compute the expected minimum distortion $\bar{D}_{k}=\bar{D}_{k-1}+D_{k} \cdot\left(\tilde{P}_{L}(k+1)-\tilde{P}_{L}(k)\right)$ and retain only the path associated with the lowest cumulative expected distortion up to that stage.

3. Assume that the bits in the first $k$ blocks are determinate and calculate $\tilde{P}_{L}$ using the estimated erasure probabilities given in Fig. 4.

4. Set $k=k+1$. If $k<K$, go to step 2 .

5. $\bar{D}_{K}=\bar{D}_{K-1}+\left(1-\tilde{P}_{L}(K)\right) \cdot D_{K}$.

6. Allocate the RS protections $Q(k), k=1, \ldots, K$ that correspond to the path with the overall lowest cumulative expected distortion.

\section{EXPERIMENTAL RESULTS}

The proposed schemes were experimentally evaluated for the transmission of the $512 \times 512$ test image "Lena" over BSC and GE channels. The scalable bitstream was generated using the SPIHT source coder. The considered transmission scenarios were over BSC with BER 0.1 and over the GE channels described in [14].

Irregular LDPC codes generated using the algorithm in [20] were employed. Two LDPC codes were used with the proposed scheme: the $(1904,4136)$, which employs packets of 517 bytes, and the $(3880,8272)$ with packets of 1034 bytes. The maximum allowable number of LDPC iterations was set to 50. The RS codes utilized by the proposed scheme are maximum separable distance (MDS) codes defined on $G F\left(2^{8}\right)$. The performance of the proposed technique was improved using an S-random interleaver with $S=15$. The interleaver parses the bits recovered during product coding in the systematic part of the packets. This process improves the overall performance of the scheme and allows faster and more effective correction of the errors. It should be noted that the LDPC decoding is performed only for the packets containing errors and, usually, less than three product decoding iterations are sufficient for the correction of all errors. This is why the additional computational cost with our methodology is very small.

For the experimental evaluation of our methodologies, we report results for a UEP scheme using the optimization algorithm

\footnotetext{
${ }^{2}$ The trellis node index is omitted in our analysis for notational convenience.
}

TABLE II

Average MSE CONVERTED to PSNR OF THE Proposed SCHEME With THE METHODS IN [10], [13], and [14] FOR THE TRANSMISSION OF "LENA" FOR TRANSMISSION OVER BSC WITH BER $=0.1$

\begin{tabular}{|c||c|c|c|c|c|}
\hline $\begin{array}{c}\text { Rate } \\
\text { (in bpp) }\end{array}$ & AEC & $\begin{array}{c}{[10]} \\
\text { JPEG2000 }\end{array}$ & $\begin{array}{c}{[10]} \\
\text { SPIHT }\end{array}$ & $\begin{array}{c}{[13]} \\
\text { SPIHT }\end{array}$ & {$[14]$} \\
\hline \hline 0.252 & 30.73 & 29.92 & 30.21 & 30.68 & N/A \\
\hline 0.505 & 33.77 & 33.13 & 33.21 & 33.79 & 32.25 \\
\hline 0.994 & 36.80 & 36.03 & 36.10 & N/A & N/A \\
\hline
\end{tabular}

presented in [17], [21] (without determinate bit decoding), an enhanced scheme based on the exploitation of determinate symbols (APC), and a final scheme based on the determinate symbol exploitation and UEP using adaptively estimated error probabilities (AEC).

In Table I, the proposed APC and AEC approaches of Section IV for the utilized LDPC codes were compared for transmission over a BSC channel with BER equal to 0.1. All the reported mean PSNR values are computed by averaging decoded MSE values and then converting the mean MSE to the corresponding PSNR value. All reported results are averages over 10000 simulations. It can be seen that the AEC approach is significantly better than the APC. The main reason is that the APC algorithm, although it employs the same product coding scheme as the AEC, in general, tends to overprotect the source information since it does not take into account the iterative product decoding and its impact on erasure probabilities. From Table I, it can also be easily deduced that the packet length affects the performance of the proposed schemes. In general, LDPC decoding is more efficient when applied to large packets. Therefore, in order to achieve the same packet erasure probabilities under the same channel conditions, LDPC codes with higher channel code rates can be used when the packets are larger; that leads to more decodable source rate.

In Table II, the best performing of the above schemes, i.e., the AEC approach using $(3880,8272)$ LDPC coder, was compared to the methods in [10], ${ }^{3}$ [13], and [14] for transmission over a BSC channel with BER equal to 0.1. As seen, the proposed method outperforms the other methods in the comparison apart from the method in [13] with which it has equivalent performance. Specifically, the proposed method outperforms the method in [14] by approximately $1.5 \mathrm{~dB}$ at $0.505 \mathrm{bpp}$. It also achieves better performance than [13] at $0.252 \mathrm{bpp}$ while it is slightly worse at $0.505 \mathrm{bpp}$. However, it is worth noting that the method in [13] uses larger packets (the codewords are as long as the entire transmitted stream). Due to the larger packets,

\footnotetext{
${ }^{3}$ The scheme in [10] employs packets of 517 bytes.
} 
TABLE III

Average MSE Converted to PSNR OF THE Proposed SCHEME With THE METHODS IN [14] FOR THE TRANSMISSION OF "LENA" CODED AT 0.50 bpp OVER A VARIETY OF GE CHANNELS

\begin{tabular}{|c||c|c|c|c|}
\hline GE channel & UEP & APC & AEC & {$[14]$} \\
\hline \hline channel1 & 32.54 & 32.65 & 33.77 & 32.25 \\
\hline channel2 & 32.54 & 32.65 & 33.77 & 32.22 \\
\hline channel3 & 32.54 & 32.65 & 33.77 & 32.24 \\
\hline channel4 & 32.54 & 32.65 & 33.77 & 32.26 \\
\hline
\end{tabular}

more decoding iterations are required. According to the authors of [13], this fact results in approximately double complexity in comparison to their method in [10], [22]. It should be noted that both the proposed scheme and the method in [10] use the logarithmic version of SPA. Moreover, the maximum number of SPA iterations in our scheme is 50, while, in [10], up to 80 iterations are allowed. Considering the minimal additional computational cost which is introduced by our iterative product decoding (usually only a few packets are needed to be redecoded), we conclude that our scheme and the scheme in [10] are of approximately equal complexity whereas the complexity of our scheme is lower than that of the method in [13]. Finally, the latency problem is less pronounced in our scheme since the decoding of packets that are not erased can start immediately provided that no other packets have been erased previously. In [13], the decoding starts only after the whole bitstream is received.

The proposed schemes were also evaluated for transmission over the GE channels used in [14]. Comparisons of our AEC method with the best method in [14], presented in Table III, demonstrate considerable performance improvements of approximately $1.50 \mathrm{~dB}$.

\section{CONCLUSION}

We proposed a novel scheme for error-resilient image transmission. The proposed scheme is based on a combination of a new technique for the exploitation of determinate symbols in Tanner graph decoding of LDPC codes and a novel product code optimization technique based on error estimation. Experimental evaluation demonstrated the superiority of the proposed system in comparison to recent state-of-the art techniques for image transmission.

\section{REFERENCES}

[1] A. Said and W. A. Pearlman, "A new fast and efficient image codec based on set partitioning in hierarchical trees," IEEE Trans. Circuits Syst. Video Technol., vol. 6, no. 3, pp. 243-250, Jun. 1996.

[2] JPEG2000 Part I Final Draft International Standard, ISO/IEC JTC 1/SC 29/WG1, Doc. No. N1855, 2000.

[3] T. Wiegand, G. J. Sullivan, G. Bjntegaard, and A. Luthra, "Overview of the H.264/AVC video coding standard," IEEE Trans. Circuits Syst. Video Technol., vol. 13, no. 7, pp. 560-576, Jul. 2003.

[4] G. Sherwood and K. Zeger, "Error protection for progressive image transmission over memoryless and fading channels," IEEE Trans. Commun., vol. 46, no. 12, pp. 1555-1559, Dec. 1998.

[5] J. Hagenauer, "Rate-compatible punctured convolutional codes (RCPC codes) and their applications," IEEE Trans. Commun., vol. 36, no. 4, pp. 389-400, Apr. 1989.
[6] S. Lin and D. J. Costello, Error Control Coding: Fundamentals and Applications. Englewood Cliffs, NJ: Prentice-Hall, 1983.

[7] V. Chande and N. Farvardin, "Progressive transmission of images over memoryless noisy channels," IEEE J. Sel. Areas Commun., vol. 18, no. 6, pp. 850-860, Jun. 2000.

[8] V. Stankovic, R. Hamzaoui, and Z. Xiong, "Fast algorithm for optimal error protection of embedded wavelet codes," in Proc. IEEE Workshop on Multimedia Signal Processing, Cannes, France, Oct. 2001, pp. 593-598.

[9] N. V. Boulgouris, N. Thomos, and M. G. Strintzis, "Transmission of images over noisy channels using error-resilient wavelet coding and forward error correction," IEEE Trans. Circuits Syst. Video Technol., vol. 13, no. 12, pp. 1170-1181, Dec. 2003.

[10] C. Lan, T. Chu, K. R. Narayanan, and Z. Xiong, "Scalable image and video transmission using irregular repeat-accumulate codes with fast algorithm for optimal unequal error protection," IEEE Trans. Commun., vol. 52, no. 7, pp. 1092-1101, Jul. 2004.

[11] H. Jin, A. Khandekar, and R. M. McEliece, "Irregular repeat accumulate codes," in Proc. 2nd Int. Symp. Turbo Codes, Brest, France, Sep. 2000, pp. 1-8.

[12] B. A. Banister, B. Belzer, and T. R. Fischer, "Robust image transmission using JPEG2000 and turbo-codes," IEEE Signal Process. Lett., vol. 9, no. 4, pp. 117-119, Apr. 2002.

[13] C. Lan, K. R. Elefteriou, and Z. Xiong, "Source optimized irregular repeat accumulate codes with inherent unequal error protection capabilities and their application to image transmission," in Proc. 37th Asilomar Conf. Signals, Pacific Grove, CA, Nov. 2003, vol. 2, pp. 1505-1509.

[14] L. Cao and C. W. Chen, "A novel product coding and recurrent alternate decoding scheme for image transmission over noisy channels," IEEE Trans. Commun., vol. 51, no. 9, pp. 1426-1431, Sep. 2003.

[15] R. Gallager, "Low-density parity-check codes," IRE Trans. Inf. Theory, pp. 21-28, Jan. 1962.

[16] D. Divsalar and F. Pollara, "Turbo codes for pcs applications," in Proc. IEEE Int. Conf. Communication, Seattle, WA, Jun. 1995, pp. 54-59.

[17] N. Thomos, N. V. Boulgouris, and M. G. Strintzis, "Wireless image transmission using turbo codes and optimal unequal error protection," IEEE Trans. Image Process., vol. 14, no. 11, pp. 1890-1901, Nov. 2005.

[18] R. M. Tanner, "A recursive approach to low complexity codes," IEEE Trans. Inf. Theory, vol. 27, no. 5, pp. 533-547, Sep. 1981.

[19] W. E. Ryan, "An introduction to LDPC codes," in CRC Handbook for Coding and Signal Processing for Recording Systems, B. Vasic, Ed. Boca Raton, FL: CRC, 2004.

[20] X.-Y Hu., E. Elefteriou, and D. M. Arnold, "Progressive edge-growth tanner graphs," in Proc. IEEE Global Telecommunications Conf., San Antonio, TX, Nov. 2001, vol. 2, pp. 995-1001.

[21] N. Thomos, N. V. Boulgouris, and M. G. Strintzis, "Wireless image transmission using turbo codes and optimal unequal error protection," in Proc. IEEE Int. Conf. Image Processing, Barcelona, Spain, Sep. 2003, pp. 73-76.

[22] C. Lan, K. R. Narayanan, and Z. Xiong, "Scalable image and video transmission using irregular repeat-accumulate (IRA) codes," in Proc. IEEE Int. Conf. Image Processing, Rochester, NY, Sep. 2002, pp. $717-720$.

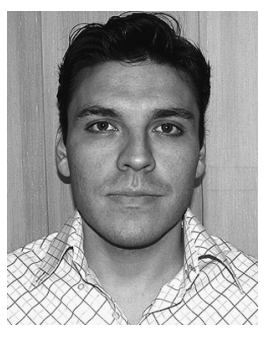

Nikolaos Thomos (S'02-M'06) received the Diploma and Ph.D. degrees from the Electrical and Computer Engineering Department, Aristotle University of Thessaloniki, Thessaloniki, Greece, in 2000 and 2005, respectively.

During his studies, he held teaching and research assistantship positions with the Electrical and Computer Engineering Department, Aristotle University of Thessaloniki. He was also Postgraduate Research Fellow with the Informatics and Telematics Institute, Thessaloniki, where he is currently a Postdoctoral Research Fellow. His research interests include joint source and channel coding, image/video coding and transmission, multimedia networking, distributed source coding, space time coding, and digital filters.

Dr. Thomos is a member of the Technical Chamber of Greece. 


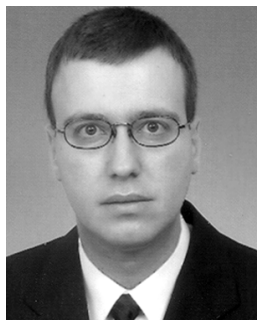

Nikolaos V. Boulgouris (S'96-M'04) received the Diploma and Ph.D. degrees from the Electrical and Computer Engineering Department, University of Thessaloniki, Greece, in 1997 and 2002, respectively.

Since December 2004, he has been a Lecturer with the Department of Electronic Engineering, Division of Engineering, King's College, London, U.K. From September 2003 to November 2004, he was a Postdoctoral Fellow with the University of Toronto, Toronto, ON, Canada. Previously, he was with the Informatics and Telematics Institute, Greece. He has participated in several research projects in the areas of image/video communication, pattern recognition, multimedia security, and content-based indexing and retrieval.

Dr. Boulgouris is a member of the British Machine Vision Association.

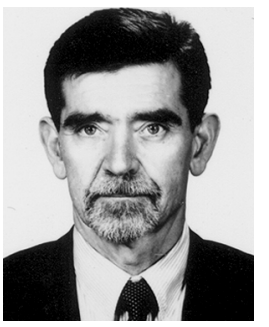

Michael G. Strintzis (S'68-M'70-SM'80-F'04) received the Diploma in electrical engineering from the National Technical University of Athens, Athens, Greece, in 1967 and the M.A. and Ph.D. degrees in electrical engineering from Princeton University, Princeton, NJ, in 1969 and 1970, respectively.

He joined the Electrical Engineering Department, University of Pittsburgh, Pittsburgh, PA, where he served as an Assistant Professor from 1970 to 1976 and an Associate Professor from 1976 to 1980. During that time, he worked in the area of stability of multidimensional systems. Since 1980, he has been a Professor of electrical and computer engineering at the Aristotle University of Thessaloniki, Thessaloniki, Greece. He has worked in the areas of multidimensional imaging and video coding. Over the past ten years, he has authored over 100 journal publications and over 200 conference presentations. In 1998, he founded the Informatics and Telematics Institute, currently part of the Centre for Research and Technology Hellas, Thessaloniki.

Dr. Strintzis was awarded the Centennial Medal of the IEEE in 1984 and the Empirikeion Award for Research Excellence in Engineering in 1999. 